Anjou. Maine. Poitou-Charente. Touraine

118-3 | 2011

La naissance de l'archéologie régionale dans l'Ouest armoricain

\title{
La Société Polymathique et la naissance de collections archéologiques en Morbihan
}

The Polymatic Society and the birth of the morbihan collections

\section{Christophe Le Pennec}

\section{Q OpenEdition}

Journals

\section{Édition électronique}

URL : http://journals.openedition.org/abpo/2055

DOI : $10.4000 / a b p o .2055$

ISSN : 2108-6443

\section{Éditeur}

Presses universitaires de Rennes

Édition imprimée

Date de publication : 30 septembre 2011

Pagination : 73-96

ISBN : 978-2-7535-1770-7

ISSN : 0399-0826

\section{Référence électronique}

Christophe Le Pennec, "La Société Polymathique et la naissance de collections archéologiques en Morbihan », Annales de Bretagne et des Pays de l'Ouest [En ligne], 118-3 | 2011, mis en ligne le 30 novembre 2013, consulté le 19 avril 2019. URL : http://journals.openedition.org/abpo/2055 ; DOI : $10.4000 / a b p o .2055$ 


\title{
La Société Polymathique et la naissance de collections archéologiques en Morbihan
}

\author{
Christophe LE PENNEC \\ Musée de Vannes
}

Les collections d'un musée, quel qu'il soit, sont le fruit de la passion des hommes, une passion pour l'art, l'archéologie ou les sciences. Dans le département du Morbihan, deux institutions possèdent aujourd'hui des collections d'archéologie régionale majeures : le musée de Préhistoire de Carnac et la Société Polymathique du Morbihan. La constitution de ces collections est intimement liée à des recherches de terrain menées par des érudits locaux, principalement durant le XIX ${ }^{\mathrm{e}}$ siècle, ainsi qu'à d'innombrables découvertes isolées.

Sous l'Ancien Régime, les prémices de ces futures collections naissent dans les cabinets de curiosités de quelques aristocrates fortunés ou savants. En Bretagne, un personnage illustre cette époque : ChristophePaul de Robien (1698-1756), président à mortier du parlement de Bretagne ${ }^{1}$. Possédant une demeure à Locmariaquer, il s'intéresse tout naturellement aux antiquités du Morbihan et collecte divers objets archéologiques, mais aussi de nombreux spécimens naturels. Au début du XIX ${ }^{\mathrm{e}}$ siècle, seuls quelques antiquaires explorent les monuments anciens, dans le seul but d'enrichir leurs collections personnelles. C'est ainsi qu'en juillet 1811, des particuliers d'Auray fouillent un dolmen à Locmariaquer sous la direction d'un certain Monsieur Renaud. Le butin récolté est mince, mais comprend tout de même une pelote de fils d'or. Cette découverte est relatée par Armand Maudet de Penhouët en $1814^{2}$.

1. AUBERT, Gauthier, Le Président de Robien : gentilhomme et savant dans la Bretagne des lumières, Rennes, PUR, 2001, 396 p.

2. Recherches historiques sur la Bretagne, chez Victor Mangin, Nantes, 1814, 139 p. Dès le début du XIX ${ }^{\mathrm{e}}$ siècle, cet érudit s'est particulièrement intéressé aux monuments mégalithiques de Carnac et de Locmariaquer. 


\section{6 : Naissance de la Société Polymathique du Morbihan, un acte fondateur}

La décennie 1820-1830 marque un tournant important dans le Morbihan, avec la publication de deux ouvrages d'érudition : Essai sur les antiquités du département du Morbihan par le chanoine Joseph Mahé (1825) ${ }^{3}$ et Les antiquités de la Bretagne par le chevalier de Fréminville $(1827)^{4}$ (fig. 1).

Mais l'évènement le plus important est la création de la Société Polymathique du Morbihan, le 29 mai $1826^{5}$. Fondée par une quinzaine de notables ou d'érudits, dont l'abbé Mahé, cette société savante se fixe "pour objet de ses études les sciences, les arts et la philologie. Elle s'occupe également de réunir dans un Musée diverses productions naturelles, surtout celles du Morbihan, des produits intéressans des arts et d'autres objets curieux de plusieurs genres" (art. Ir de son règlement intérieur).

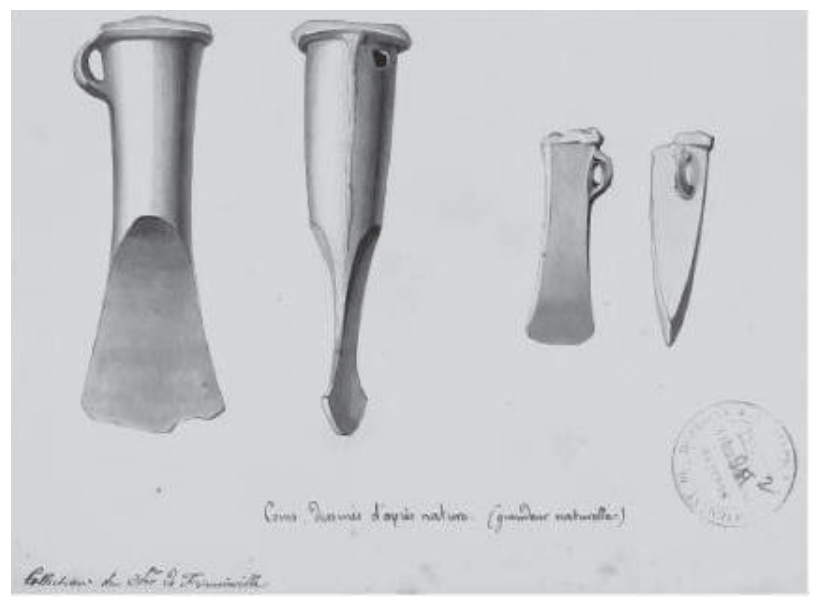

Figure 1 -

Haches à douilles de la collection du chevalier de Fréminville (dessin, XIXe siècle (C) Manuscrits de la Société Polymathique du Morbihan)

Un de ses membres, M. Rallier, a représenté les avantages que le Morbihan doit retirer de l'érection d'un musée tel qu'il est envisagé, et développé son propos sur l'intérêt de fouilles bien menées : "Ces fouilles, dit notre honorable confrère, vous présentent les plus belles chances de succès, sous le triple point de vue que, sans leur secours, la partie minéralogique de la collection projetée demeure incomplète; qu'elles peuvent conduire à

3. Essai sur les antiquités du département du Morbihan, imp. Galles aîné, Vannes, 1825, 500 p., 5 pl. gr. Antiquités de la Bretagne, chez Lefournier et Deperiers, libraires, Brest, $1827,40 \mathrm{p}$.

4. Ibidem.

5. Pour mieux connaître l'histoire de cette société savante : Centenaire de la Société Polymathique du Morbihan (1826-1926), Vannes, Lafolye frères et Cie éditeurs, 1927, 251 p. FRÉLAUT, Bertrand, " Les grandes heures de la Société Polymathique du Morbihan (18262006) ", BSPM, 2006, CXXXII, p. 181-267. 
des découvertes archéologiques importantes; enfin, par-dessus tout, qu'elles doivent un jour nous révéler de riches productions naturelles cachées sous nos pas, et dont l'exploitation sera une source d'abondance pour le département $^{6}$."

La naissance de cette société savante installe déjà les principes qui vont marquer le Morbihan durant tout le XIX ${ }^{\mathrm{e}}$ siècle : des recherches scientifiques collégiales et la création d'un musée pour conserver et présenter au public les divers éléments collectés. L'un des fondateurs, Jean-Marie Galles, botaniste et minéralogiste, appartient aussi à la sixième génération de la célèbre dynastie d'imprimeurs vannetais. Cette famille va largement contribuer aux travaux de la Société Polymathique du Morbihan et permettre la publication de ses bulletins.

\section{6-1846 : L'archéologie, une activité oubliée ou presque}

Depuis déjà plusieurs années, quelques botanistes avaient pour habitude de se réunir pour herboriser dans les environs de Vannes. Avec la fondation de la société savante, cette démarche devient réellement scientifique et s'étend à toutes les espèces des trois règnes de la nature : végétal, animal et minéral. La collecte d'échantillons, d'abord limitée au Morbihan, s'étend ensuite à toutes les régions du globe et conduit à la constitution d'une remarquable collection.

Les collections naturelles du musée de Vannes résultent à la fois des travaux de quelques érudits locaux (Jean-Marie Galles, Amand Taslé, Emmanuel Le Lièvre, etc.) et de centaines de donations de spécimens, rapportés de voyages à l'étranger par des particuliers. Ces voyageurs sont souvent des marins, civils ou militaires, en fonction dans les territoires français d'outremer et bon nombre sont originaires de Bretagne. C'est ainsi que le musée de Vannes, comme d'autres de la région, voit sa collection s'enrichir aussi de centaines d'objets exotiques, artefacts de civilisations lointaines ou disparues ${ }^{7}$.

À cette époque, la Société Polymathique se préoccupe déjà de la protection des antiquités du Morbihan. Le 6 février 1828, à la demande du chanoine Mahé, le comte de Chazelles (préfet du Morbihan) envoie une circulaire à tous les maires de son ressort pour leur prescrire de verbaliser " contre tous les individus qui tenteraient de mutiler ou de s'emparer des monuments celtiques qui peuvent exister dans votre commune... ". Après le décès de Mahé (1831), seuls deux nouveaux membres s'intéressent aux antiquités morbihannaises : Gaillard et Cayot-Delandre. Pourtant, en 1832, la société savante n'est pas informée par le préfet du dégagement du célèbre monument de Gavrinis. Très peu de fouilles archéologiques sont réalisées par les polymathes et seules quelques trouvailles isolées sont à signaler :

6. Compte rendu des travaux de la Société Polymathique du Morbihan pendant l'année 1826-1827, Vannes, imp. Galles, 1827, p. 6.

7. LE PENNEC, Christophe, "Les collections extra-européennes du musée de Vannes ", BSPM, 2009, CXXXV, p. 377-388. 
- 1835 : découverte de la borne milliaire de Liscorno à Surzur (achetée par la Société Polymathique du Morbihan en 1853).

- 1842 : découverte d'une borne milliaire et fouilles d'une villa galloromaine à Saint-Christophe à Elven, par le capitaine Le Thann (des objets sont donnés à la Société Polymathique du Morbihan et celle-ci achète la borne).

- 1842 : fouilles de vestiges antiques près de Saint-Symphorien à Vannes, par Jean-Marie-Galles (découverte restée inédite jusqu'en 1960) ${ }^{8}$.

En 1843, un évènement vient notablement changer la donne dans la cité vannetaise : c'est la création de l'Association Bretonne par un groupe d'agronomes et de propriétaires terriens. Dès 1846, elle ouvre une section d'archéologie présidée par Amand Taslé et vient concurrencer directement la Société Polymathique du Morbihan, tombée peu à peu en léthargie ${ }^{9}$.

\section{6-1859 : L’Association Bretonne, section antiquités et archéologie}

Vers le milieu du XIX ${ }^{\mathrm{e}}$ siècle, les recherches archéologiques sont donc le fruit de deux institutions distinctes : la section archéologique nouvellement crée par l'Association Bretonne, et la $4^{\mathrm{e}}$ section de la Société Polymathique, intitulée "antiquités et archéologie". Pourtant, les chercheurs vannetais sont si peu présents sur le terrain que l'on assiste ici et là à des initiatives individuelles hasardeuses qu'on pourrait parfois qualifier de pillages. En 1849, le maire de Plouharnel, M. Le Bail, décide d'explorer le lieu appelé alors "les grottes de Rondossec ". Ce monument, qui s'avère être un tumulus à trois dolmens, est dégagé sans méthode et les objets découverts sont brisés ou dispersés ${ }^{10}$.

Dans ce contexte, il devenait de plus en plus inconcevable d'avoir à Vannes deux sociétés traitant d'un même domaine de recherche, d'autant que les mêmes personnes y siégeaient. En 1853, un compromis semble trouvé par la création de la Société Archéologique du Morbihan, nouvelle section plus ou moins autonome de la Société Polymathique du Morbihan. Certaines personnalités qui la composent vont contribuer activement aux futures recherches : Amand Taslé ${ }^{11}$, François-Marie Cayot-Delandre ${ }^{12}$,

8. Bibliothèque de la SPM, ms. 52. ANDRÉ, Jacques, " Une villa inédite entourée d'une enceinte à Vannes ", OGAM, 1960, p. 169-173.

9. Centenaire de la Société Polymathique du Morbihan (1826-1926), op. cit., p. 80-82.

10. Closmadeuc, Gustave de, "La découverte des grottes de Plouharnel ", BSPM, 1882, 26, p. 174-178. Les objets découverts, qui ont été récupérés par des particuliers, comprenaient deux célèbres colliers ou brassards en or.

11. Amand Taslé (1801-1876), l'un des fondateurs de la Société Polymathique du Morbihan, a fait une carrière de notaire. Par son mariage, il se retrouve lié à une famille de notables bretons qui comprend des préfets et des maires. Il a été neuf fois président de la Société Polymathique, dans les années 1838-1859, y compris quand il est maire de Vannes (1839-1846). Botaniste et naturaliste, il est conservateur-adjoint de Jean-Marie Galles de 1826 à 1860 et lui succède jusqu'à sa mort en 1876. Il a en grande partie réalisé l'herbier départemental de la Société Polymathique du Morbihan. À sa mort, il lègue au musée sa collection d'histoire naturelle.

12. Le Morbihan, son histoire, ses monuments, Vannes, imp. Cauderan, 1847. 
Louis Galles ${ }^{13}$, Alfred Fouquet ${ }^{14}$ et surtout deux ingénieurs des Ponts et Chaussées, Jacquemet et de Fréminville.

Cette situation nouvelle permet une effervescence dans la recherche archéologique et est suivie d'effets positifs majeurs : la création d'un musée archéologique (1853) et la reprise des publications (1857). Les premières fouilles menées par les uns et les autres, sont désormais plus rigoureuses et conduisent à déjà d'importantes découvertes de vestiges et d'objets :

- En 1853, Alfred Fouquet explore le tumulus carnacéen de Tumiac à Arzon, et recueille un important mobilier funéraire néolithique : 32 haches polies (jadéite, chloromélanite, fibrolite), 237 perles et 12 pendeloques en variscite $^{15}$. La même année, il publie un ouvrage intitulé Des monuments celtiques et des ruines romaines dans le Morbihan ${ }^{16}$.

- En 1854, Jean-Marie Galles découvre dans sa propriété de Saint-Galles à Arradon, un mobilier funéraire de l'âge du Fer composé de nombreux bracelets à bossettes en bronze, de fragments d'autres bracelets (fer, lignite) et de perles.

- En 1856, M. de La Fruglaye fouille le dolmen de Le Resto à Moustoirac et recueille un très beau poignard en silex du Grand-Pressigny, de $24,8 \mathrm{~cm}$ de longueur.

Entre 1856 et 1858, les ingénieurs de Fréminville, Jacquemet et Grégoire, apportent leur contribution, en dressant le plan précis de plusieurs établissements gallo-romains : Saint-Symphorien à Vannes, Le Lodo (fig. 2) et Mané Bourgerel à Arradon, Tréalvé à Saint-Avé, etc. Ils mettent aussi au net les plans de découvertes plus anciennes telle que la villa Saint-Christophe à Elven. La production scientifique devient si importante que les bulletins annuels ne permettent plus de tout publier. Depuis lors, cette documentation archéologique et historique, en partie inédite, alimente les archives de la Société Polymathique et constitue aujourd'hui le fonds des manuscrits (environ 2000 références) ${ }^{17}$.

Dans la plupart des cas, le mobilier archéologique découvert est donné ou déposé à la Société Polymathique, qui possède désormais deux musées (archéologie et sciences naturelles) sous la direction d'un seul conservateur. Un récapitulatif de l'enrichissement des collections est publié en fin de chaque bulletin annuel. En complément du mobilier issu des fouilles, divers autres dons sont mentionnés : objets historiques, trouvailles iso-

13. Louis Galles (1827-1874), imprimeur-libraire à Vannes, fils de Jean-Marie, l'un des fondateurs de la Société Polymathique du Morbihan.

14. Solary-JaVAudin, Catherine, Le Docteur Alfred Fouquet : un notable vannetais au $X I X^{e}$ siècle, Université Catholique de l'Ouest (UCO), Angers, 1997.

15. Rapport sur la découverte d'une grotte sépulcrale dans la butte de Tumiac, le 21 juillet 1853, imp. Cauderan, Vannes, 1853. Ce rapport a été adressé au préfet du Morbihan, le $1^{\text {er }}$ août 1853, au nom de la Société Polymathique du Morbihan.

16. Chez A. Cauderan, libraire-éditeur, Vannes, 1853, 117 p.

17. LE PENNEC, Christophe, "Inventaire informatisé des manuscrits anciens (premier bilan) ", Bulletin mensuel de la Société Polymathique du Morbihan, n 1678, septembre 2004, p. 83 . 


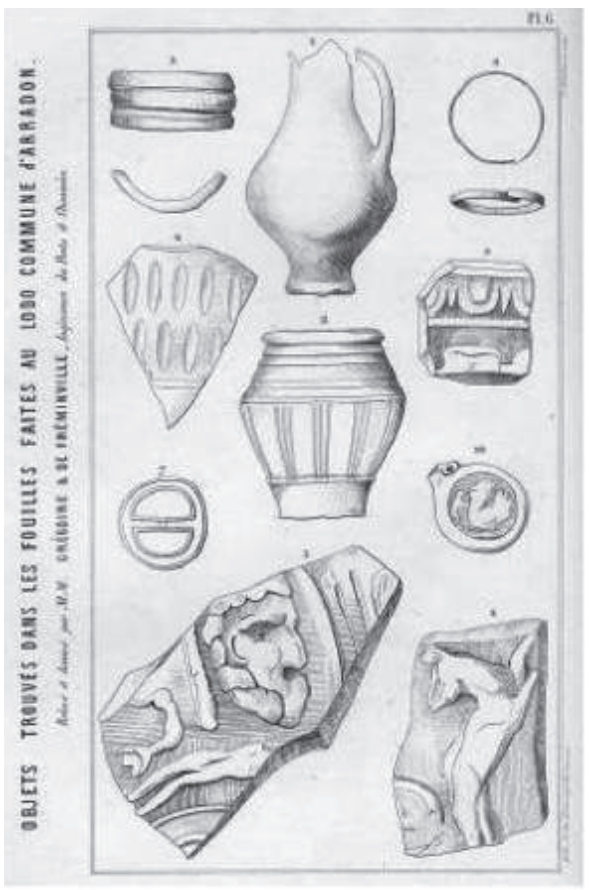

Figure 2 - Objets galloromains de la villa du Lodo (lithographie d'après les dessins de Grégoire et de Fréminville, BSAM, 1857)

lées, et nombreuses monnaies et médailles de toutes époques et de toutes provenances $^{18}$.

L'année 1859 est marquée par la dissolution de l'Association Bretonne, décidée par le gouvernement de Napoléon III, sans doute pour des raisons politiques. Cela ne change finalement pas grand-chose à la situation puisque ses membres et activités étaient communs avec la Société Polymathique, désormais seule. Un nouveau sociétaire fait aussi son entrée : le jeune Gustave de Closmadeuc (1828-1918) ${ }^{19}$. Personnage incontournable de la seconde moitié du XIX ${ }^{\mathrm{e}}$ siècle, il consacre l'essentiel de son temps libre à sa passion, l'archéologie. Il prend part à de nombreuses fouilles et publie des dizaines d'articles scientifiques. En cette fin de décennie, tous les ingré-

18. Au cours des décennies suivantes, toutes ces monnaies vont peu à peu constituer un imposant médaillier.

19. Né à La Roche-Bernard, il a suivi les traces de son père, médecin des épidémies. Encore jeune étudiant, il se distingue dans la lutte contre les épidémies de choléra et de dysenterie qui frappent la France entre 1849 et 1852. Devenu chirurgien, il réalise quelques prouesses médicales (opérations césariennes) et s'implique dans l'amélioration de l'accès aux soins des plus déshérités, dans la salubrité publique et les hôpitaux de Vannes. Pour en savoir plus sur cet érudit : Audic, Marie Laure, Le Docteur de Closmadeuc, médecin, archéologue et historien, mémoire de maîtrise d'histoire contemporaine, UCO, Angers, 1997, 213 p. ARs, François, " De Closmadeuc, médecin et archéologue ", BSPM, 1999, CXXV, p. 249-256. 
dients sont désormais réunis pour permettre à la recherche archéologique dans le Morbihan de connaître un véritable essor.

Figure 3 - Amand Taslé, Gustave de Closmadeuc et René Galles (photographies et dessin)

(musée de Vannes, fonds Société Polymathique du Morbihan)
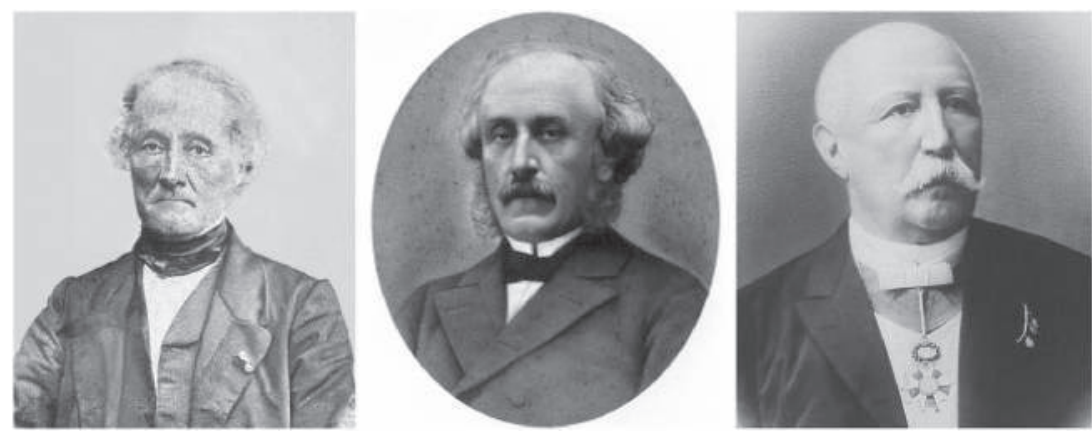

1860-1914 : Un âge d'or pour la recherche archéologique en Morbihan

\section{La découverte de très nombreux vestiges}

Les fouilles menées par la Société Polymathique sont si nombreuses qu'il est désormais impossible de toutes les énumérer. Sur cinquante ans, plus d'une centaine de sites sont en effet explorés :

\begin{tabular}{|c|c|}
\hline Période & $\begin{array}{c}\text { Nombre approximatif de fouilles réalisées } \\
\text { par la Société Polymathique du Morbihan }\end{array}$ \\
\hline $1860-1870$ & 52 (dont 22 en 1866) \\
\hline $1871-1880$ & 21 \\
\hline $1881-1890$ & 22 \\
\hline $1891-1900$ & 2 \\
\hline $1901-1914$ & 5 \\
\hline
\end{tabular}

De 1860 à 1865, les recherches de terrain sont généralement l'œuvre de René Galles et son cousin Louis Galles, aidés de Julien Lefebvre, préfet du Morbihan, et d'Alphonse Mauricet. Ils explorent ainsi les fameux tumulus carnacéens de Saint-Michel (1862) et du Moustoir (1864) à Carnac, et de Mané-er-Hroëck (1863) à Locmariaquer; auxquels il faut ajouter d'autres tumulus néolithiques : Kercado à Carnac (1863), Mané-Lud à Locmariaquer (1863) et le Petit-Mont à Arzon (1865) ${ }^{20}$. De son côté, Gustave

20. «Les fouilles du Mont-Saint-Michel en Carnac », BSPM, 1862, 6, p. 7-44. "Fouilles du tumulus du Moustoir, Carnac ", BSPM, 1864, 8, p. 117-125. "Un dolmen découvert sous la 
de Closmadeuc consacre son temps libre à la fouille de plusieurs dolmens et s'intéresse déjà aux nécropoles protohistoriques dites " à coffres de pierres $^{21}$ ". Si presque toutes les fouilles menées concernent des monuments funéraires néolithiques, Louis Galles explore néanmoins l'établissement gallo-romain de Kerhan à Arradon ${ }^{22}$. Une découverte isolée ne passe pas inaperçue et vient enrichir le musée archéologique : le dépôt métallique du Parc-aux-Bœufs à Questembert, composé de 30 kg d'objets ou fragments de bronze ${ }^{23}$ (fig. 4 et 5). En 1866 et 1867, les recherches se concentrent sur les sites néolithiques du pays d'Auray. Un nouveau polymathe collabore aux fouilles : Antoine Léon Davy de Cussé (1823-1886) ${ }^{24}$. Par le nombre de monuments explorés, cette période est sans doute la plus riche.

\begin{tabular}{|c|c|c|c|c|}
\hline \multicolumn{5}{|c|}{ Tableau des fouilles réalisées en 1866 et 1867 par la Société Polymathique } \\
\hline Commune & Nom du site & Fouille menée par & $\begin{array}{l}\text { Nb objets } \\
\text { (environ) }\end{array}$ & $\begin{array}{c}\text { Mode d'entrée au } \\
\text { musée }\end{array}$ \\
\hline Arzon & $\begin{array}{l}\text { Tumulus de } \\
\text { Bilgroez }\end{array}$ & \multirow{11}{*}{$\begin{array}{l}\text { La Société } \\
\text { Polymathique du } \\
\text { Morbihan }\end{array}$} & 40 & \multirow{11}{*}{$\begin{array}{l}\text { Mobilier archéolo- } \\
\text { gique appartenant } \\
\text { de fait à la Société } \\
\text { Polymathique du } \\
\text { Morbihan }\end{array}$} \\
\hline Arzon & îlot d'Er Lannic & & 50 & \\
\hline Carnac & $\begin{array}{l}\text { Monument de } \\
\text { Mané Clud er Yer }\end{array}$ & & 10 & \\
\hline Carnac & $\begin{array}{l}\text { Dolmen d'En } \\
\text { Autérieu }\end{array}$ & & 9 & \\
\hline Carnac & $\begin{array}{l}3 \text { dolmens de } \\
\text { Keryaval }\end{array}$ & & 38 & \\
\hline Carnac & $\begin{array}{l}\text { Monument de } \\
\text { Keryaval }\end{array}$ & & 27 & \\
\hline Carnac & $\begin{array}{l}\text { Tumulus de } \\
\text { Kervihan }\end{array}$ & & 15 & \\
\hline Carnac & $\begin{array}{l}\text { Tumulus de Mané } \\
\text { Rumentur (âge du } \\
\text { Bronze) }\end{array}$ & & 7 & \\
\hline La Trinité-sur-Mer & \begin{tabular}{|l}
2 dolmens de \\
Kervilor
\end{tabular} & & 16 & \\
\hline La Trinité-sur-Mer & Dolmen d'Er Roh & & 20 & \\
\hline Plouharnel & $\begin{array}{l}\text { Dolmen de } \\
\text { Runesto }\end{array}$ & & 5 & \\
\hline
\end{tabular}

tombelle de Kercado en Carnac ", BSPM, 1863, 7, fasc. 2, p. 5-16. " Manné-er-H'Roëk, dolmen découvert sous un tumulus ", $B S P M, 1863$, 7, fasc. 2, p. 18-32. " Note sur le Manné-Lud (Locmariaquer) ", BSPM, 1863, 7, fasc. 2, p. 33-39. Si actif durant ces trois années, René Galles ne prend plus part aux recherches archéologiques à partir de 1865, date à laquelle il devient intendant militaire hors du Morbihan.

21. "Fouille d'un dolmen tumulaire à Crubelz, et Fouilles des dolmens de Kerrock ", $B S P M, 1864,8$, p. 117-125. "Tombeau découvert au Manné-Beker-Noz (Quiberon) ", BSPM, 1865, 9, p. 39-49. Il est parfois aidé par d'autres médecins : Alphonse de Closmadeuc, M. Gressy et M. Broca.

22. BSPM, 1865, 9, p. 73-75.

23. BSPM, 1863, 7, fasc. 1, p. 10-30. Il s'agit d'un dépôt aujourd'hui daté du Bronze final 3B.

24. Propriétaire terrien, Léon de Cussé possède le manoir de Kergolher à Plaudren. En 1863, il entre à la SPM, et occupe la fonction de conservateur du musée d'Archéologie de 1866 à 1879. En 1865, il publie le Recueil des signes sculptés sur les monuments mégalithiques du Morbihan, impr. Galles, Vannes. 
La Société Polymathique et la naissance de collections archéologiques en Morbihan

\begin{tabular}{|c|c|c|c|c|}
\hline Brec'h & \begin{tabular}{|l|} 
Dolmen de \\
Kerouarin \\
\end{tabular} & \multirow{3}{*}{$\begin{array}{l}\text { Gustave de } \\
\text { Closmadeuc }\end{array}$} & 2 & \multirow{4}{*}{ Don du fouilleur } \\
\hline Carnac & Dolmen du Lizo & & 10 & \\
\hline Carnac & $\begin{array}{l}\text { Dolmen de Ker- } \\
\text { groix }\end{array}$ & & 5 & \\
\hline Crach & $\begin{array}{l}\text { Monument de Parc } \\
\text { er Gueren }\end{array}$ & $\begin{array}{l}\text { Alphonse } \\
\text { de Closmadeuc }\end{array}$ & 3 & \\
\hline La Trinité-sur-Mer & $\begin{array}{l}\text { Tumulus d'Er } \\
\text { Hourig }\end{array}$ & \multirow{6}{*}{$\begin{array}{l}\text { Léon de Cussé et } \\
\text { Louis Galles }\end{array}$} & 8 & \multirow{6}{*}{ Don des fouilleurs } \\
\hline La Trinité-sur-Mer & $\begin{array}{l}\text { Tumulus de Men } \\
\text { er Roh }\end{array}$ & & 17 & \\
\hline La Trinité-sur-Mer & $\begin{array}{l}2 \text { dolmens de } \\
\text { Kermarquer }\end{array}$ & & 3 & \\
\hline Locmariaquer & $\begin{array}{l}\text { Dolmen de Mané } \\
\text { Han }\end{array}$ & & 16 & \\
\hline Arradon & $\begin{array}{l}\text { Dolmens de la } \\
\text { Pointe du Tréh }\end{array}$ & & 10 & \\
\hline Séné & $\begin{array}{l}\text { Monuments de l'île } \\
\text { de Bouëd }\end{array}$ & & 4 & \\
\hline Plougoumelen & $\begin{array}{l}\text { Tumulus du } \\
\text { Rocher (âge du } \\
\text { Fer) }\end{array}$ & $\begin{array}{l}\text { William Collings } \\
\text { Lukis }^{\text {a }}\end{array}$ & 2 & $\begin{array}{l}\text { Achat Société } \\
\text { Polymathique du } \\
\text { Morbihan (fig. 6) }\end{array}$ \\
\hline \multicolumn{3}{|c|}{ Au moins 26 monuments fouillés sur deux ans } & \multicolumn{2}{|c|}{ Au moins 317 objets collectés } \\
\hline
\end{tabular}

a William Collings Lukis (1817-1892). "Rapport sur un tumulus de l'âge du Bronze au Rocher en Plougoumelen ", BSPM, 1867, 11, p. 110-112.

\section{Figure 4 - Objets en bronze de Questembert (Morbihan)}

(lithographie d'après un dessin du Dr de Closmadeuc, BSPM, 7, 1863)

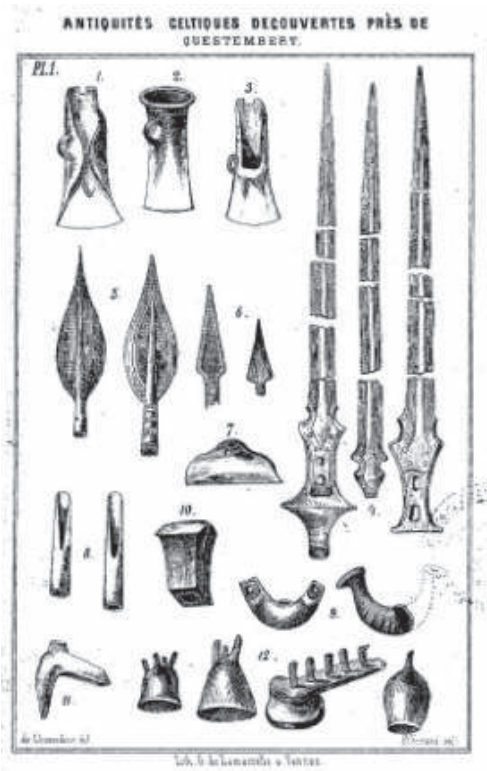

Figure 5 - Quelques objets du dépôt de Questembert (Morbihan) (photographie Hildebrand, à Vannes, vers 1865, (C) Manuscrits de la Société Polymathique du Morbihan)

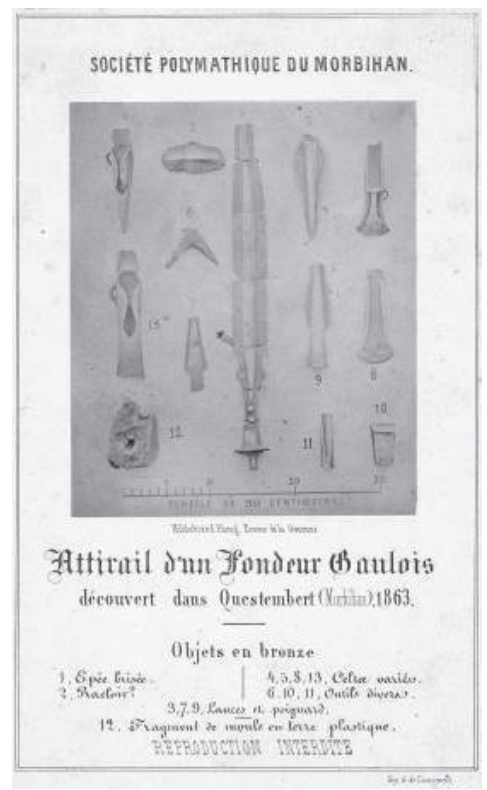


De 1868 à 1872, les fouilles sont presque toutes réalisées par des ecclésiastiques, aussi membres de la Société Polymathique du Morbihan. Les abbés Collet, Lavenot et Le Poder, explorent divers vestiges du pays d'Auray où ils exercent d'abord et surtout la fonction de curé de paroisse. Pierre Lavenot, fouille tout d'abord divers dolmens de Carnac (1869) ${ }^{25}$, puis la chapelle de Saint-Clément à Quiberon $(1870)^{26}$. Il est sans doute l'un des premiers à réaliser de véritables prospections-inventaires de sites archéologiques ${ }^{27}$. Joachim Collet, vicaire à Quiberon, relate de nombreuses observations de sites inédits. Il fouille entre autres l'éperon barré vénète de Beg-en-Aud qu'il confond malheureusement avec un tumulus néolithique et explore l'éperon néolithique de Croh-Collé28. En 1869, contraint de quitter Quiberon, il rédige une lettre à la Société Polymathique du Morbihan, où l'on peut lire ces quelques mots : "Heureusement j'ai composé un manuscrit sur toutes les fouilles opérées par moi dans Quiberon, et ce sera un souvenir que je conserverai toute ma vie, de mon séjour dans cette presqu'île ${ }^{29}$. "L'abbé Collet fait intégralement don de ses découvertes au musée archéologique de Vannes, contrairement à l'abbé Lavenot, qui en demande rétribution à la Société Polymathique du Morbihan.

De 1872 à 1886, les fouilles sont plus collégiales. Après le décès de leur père, Fernand et Henri de Cussé prennent activement part aux recherches et l'équipe est rapidement complétée par Léon Lallement et Alfred Lallemand. En 1876, lors de la construction de casernes d'artillerie, ils découvrent la nécropole antique de Vannes et recueillent près d'une centaine d'objets dont de nombreux vases intacts. Dans les dolmens de l'Île aux Moines explorés en 1877, ils trouvent au moins 50 objets. D'autres dolmens et tumulus sont découverts sur les communes de Colpo, Plumelec, Saint-Jean Brévelay et de l'île d'Arz ${ }^{30}$. Le célèbre monument néolithique de Mané Rutual, à Locmariaquer, est déblayé après avoir été racheté par l'État en 1885. Le mobilier découvert, composé d'environ 150 objets dont une centaine de fragments de figurines gallo-romaines en terre blanche,

25. "Fouilles faites par M. l'abbé Lavenot dans la commune de Carnac », BSPM, 1869, 13, p. 109-111.

26. BSPM, 1889, 33, p. 16-25. BSPM, 34, 1890, p. 164-180. Il s'agit sans doute ici d'un site majeur pour le Haut-Moyen Âge.

27. "Les îles d'Hoédic, d'Houat et la presqu'île de Quiberon ", BSPM, 1885, 29, p. 97 et $B S P M, 32,1888$, p. 106. "Hoédic, Houat et Quiberon pendant la période gallo-romaine ", $B S P M, 1888,32$, p. 183.

28. Closmadeuc, Gustave de, "Rapport sur les fouilles et découvertes de l'abbé Collet (Beg-en-Aud, Kervihan)", BSPM, 1868, 12, p. 171.

29. Collet, abbé, Quelques fouilles à St Pierre Quiberon, (lettre du 22 novembre 1869), Bibliothèque de la Société Polymathique du Morbihan, manuscrit ${ }^{\circ} 532$. On ne sait malheureusement pas ce qu'est devenu l'intéressant manuscrit que mentionne l'abbé Collet qui, en 1869, partait pour la paroisse de Saint-Aignan, près de Pontivy. En 1871, alors vicaire à Ploërmel, il fouille les sites de Mané Pleurig, Mané Bodgad et une tombelle à Kergonvo : $B S P M, 1871,15$, p. 49-50 et procès-verbaux p. 72-73.

30. Voir les nombreux articles publiés dans les BSPM, 1884 à 1886. Les trois dolmens de Pen-Liouse en lîle d'Arz sont fouillés par l'abbé Le Gouguec. 
Figure 6 - Urne en bronze trouvée à Plougoumelen (Morbihan)

en 1867 et achetée par la Société Polymathique du Morbihan

(dessin de Lukis [haut] et aquarelle de Léon de Cussé [bas], (C) Archives de la Société Polymathique du Morbihan)

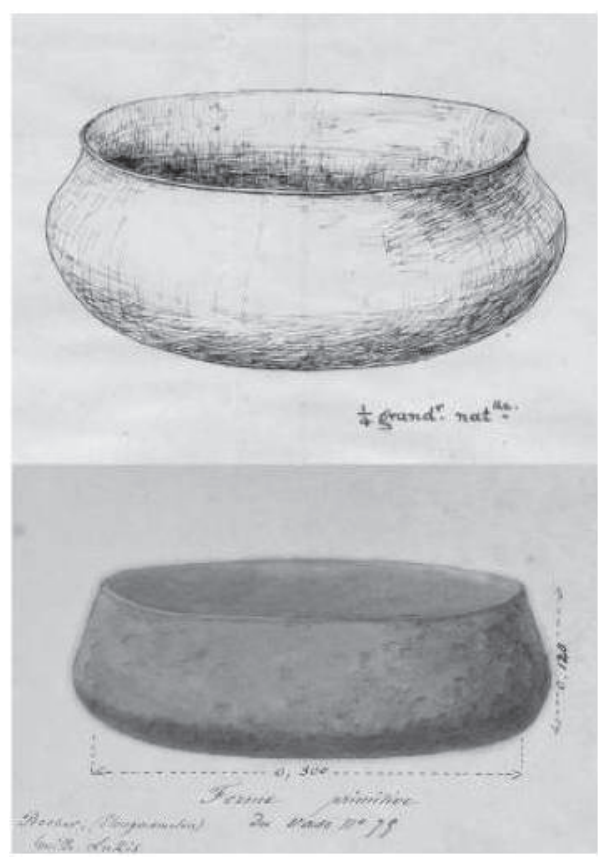

est donné à la Société Polymathique du Morbihan par le ministère des Beaux-Arts ${ }^{31}$.

De son côté, Gustave de Closmadeuc, devenu propriétaire de l'île de Gavrinis, explore à nouveau le cairn néolithique mais aussi les fondations de la ferme voisine où il exhume des sépultures médiévales ${ }^{32}$. À défaut de pouvoir énumérer toutes les trouvailles isolées, on peut évoquer les deux dépôts de bronzier mis au jour sur la commune de Guidel en 1876 et qui, à eux seuls, comprenaient une centaine d'objets ou de fragments ${ }^{33}$ (fig. 7 et cahier couleur, fig. 12).

À partir de cette époque, les polymathes concentrent surtout leurs recherches sur le pays de Vannes, car un nouveau chercheur est arrivé dans le pays d'Auray: James Miln. D'origine écossaise, après avoir longtemps voyagé en Asie, il s'installe à Carnac en 1873. Rapidement, il entreprend le dégagement de l'imposante villa gallo-romaine du

31. Ce monument avait déjà été fouillé en 1860 par le baron Arthur de Bonstetten et Louis Galles. Closmadeuc, Gustave de, "Le dolmen de Rutual (Locmariaquer) ", BSPM, 1885,29 , p. 112-119.

32. BSPM, 1885, 29, p. 134-145.

33. Très souvent, les trouvailles faites par des agriculteurs étaient signalées au curé de la paroisse ou à un notable local, propriétaire terrien. Ces deux dépôts du Bronze final ont été étudiés par l'abbé Euzenot, qui en a fait don à la Société Polymathique du Morbihan : BSPM, 1876, 20, p. 109-110. 


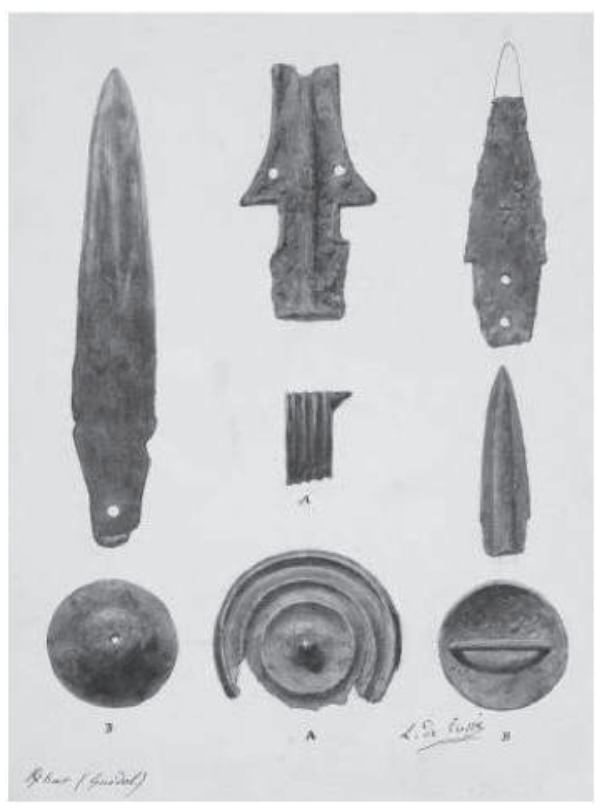

Figure 7 - Objets du dépôt de bronzier de Kerhar à Guidel (aquarelle de Léon de Cussé, vers 1870-1880,

(C) Archives de la Société

Polymathique du Morbihan)

Bossenno $^{34}$, et durant les six années qui suivent, il fouille de nombreux sites sur les communes littorales entre Erdeven et Locmariaquer (fig. 8). À son décès, survenu en 1881, son frère Robert fait construire un bâtiment pour y loger les collections archéologiques dont il vient d'hériter et fait don de l'ensemble à la commune de Carnac. C'est la naissance du musée James Miln. Un catalogue des collections, publié en 1894, relate les travaux de l'érudit écossais et comprend plus de mille numéros d'inventaire ${ }^{35}$. Dans la région de Carnac, un nouveau chercheur encore plus illustre lui succède et poursuit l'enrichissement du musée de Carnac : Zacharie Le Rouzic. Ce dernier, aussi membre de la Société Polymathique, y publiera régulièrement ses découvertes jusqu'en 1935.

De 1886 à 1914, pour plusieurs raisons, les polymathes réalisent nettement moins de fouilles archéologiques qu'auparavant. Les archéologues sont moins nombreux après une série de décès ${ }^{36}$ et même s'il poursuit encore son étude des coffres protohistoriques de la presqu'île de Quiberon (fig. 9), Gustave de Closmadeuc est désormais trop âgé. De plus, ils ne sont plus seuls dans ce domaine scientifique, comme le montre l'exemple du pays d'Auray exploré entre 1875 et les années 1930, par James Miln,

34. Miln, James, Fouilles faites à Carnac (Morbihan), Bossenno et le Mont Saint-Michel, 1877, 253 p., pl. en coul.

35. Catalogue du musée J. Miln à Carnac (Morbihan), Vannes, 1894, 51 p.

36. Décès de Louis Galles (1874), Alfred Fouquet (1875) et Léon de Cussé (1886). 
Figure 8 - Objets trouvés à Mané er Ouah-Tihir (Carnac, Morbihan) par James Miln et l'abbé Luco, en 1876, partiellement conservés aujourd'hui au musée de Carnac

(Planche aquarellée, non signée, entre 1876 et 1883, (C) Manuscrits de la Société Polymathique du Morbihan)

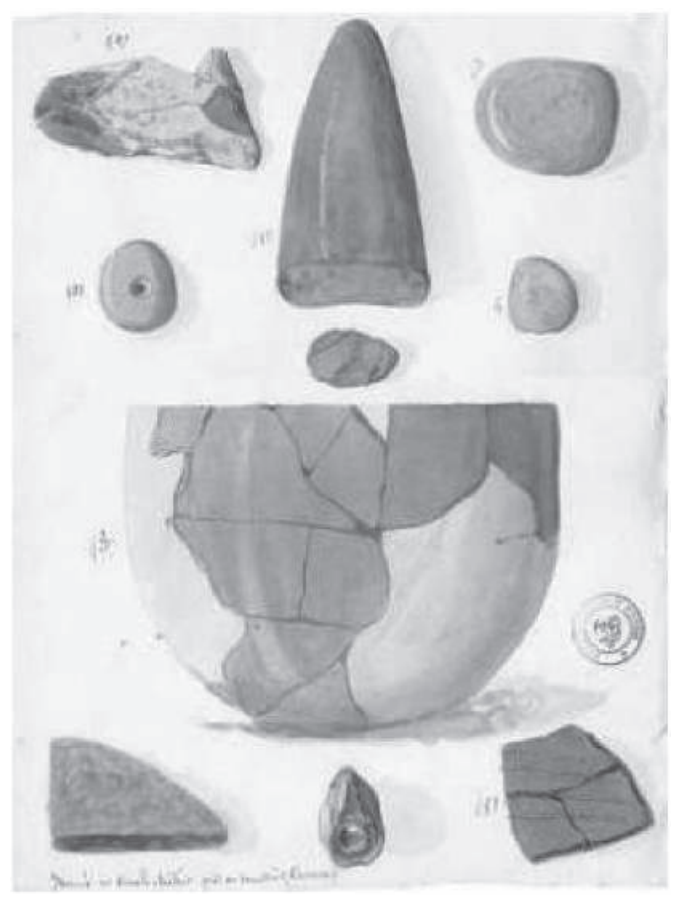

Zacharie Le Rouzic ${ }^{37}$ et Félix Gaillard ${ }^{38}$. Un autre chercheur, le vicomte Paul Aveneau de La Grancière (1862-1942), multiplie les découvertes archéologiques dans le Morbihan et rédige de nombreux articles dans les bulletins de la Société Polymathique du Morbihan ${ }^{39}$. Pourtant, aucun de ses objets exhumés dans le département ne vient enrichir le musée de Vannes, et il vend même plus de 300 éléments de sa collection au British Museum de Londres dans les années 1930.

En ce début du $\mathrm{Xx}^{\mathrm{e}}$ siècle, la priorité de la société savante est désormais la conservation des collections du musée archéologique et la recherche de locaux pour les présenter au public. La première guerre mondiale marque

37. Zacharie Le Rouzic (1864-1939). Pour découvrir l'étendue de son œuvre scientifique, cf. JACQ, Maurice, Catalogue du Musée archéologique Miln - Le Rouzic, Carnac, 1940, 255 p.

38. Issu d'une famille fortunée de la région de Bordeaux, Félix Gaillard (1832-1910) s'installe en 1857 comme propriétaire de l'Hôtel du Commerce de Plouharnel et débute ses recherches dans les années 1870 . Il réalise d'importantes fouilles, essentiellement sur Erdeven, Plouharnel et la presqu'île de Quiberon (dolmens de Port-Blanc, nécropole de Thinic, site de Beg-er-Goalennec, etc.). Ses publications, tant dans les BSPM que dans des revues nationales, sont d'une grande qualité. La majeure partie de sa collection archéologique entre par la suite au musée de Carnac. CROWHURST, Howard, Mémoire de pierres à Plouharnel (photographies et textes inédits, 1870 à 1895, de Félix Gaillard), HCom éditions, Vannes, 2004, 81 p.

39. Entre 1896 et 1911, il publie dans les BSPM, une quarantaine d'articles archéologiques dont un certain nombre consacré aux cachettes de bronziers. 
Figure 9 - Mobilier archéologique découverts à Beg-er-Vil à Quiberon (Morbihan), étudié par Gustave de Closmadeuc, et aujourd'hui conservé au musée de Vannes (BSPM, 30, 1886, p. 3-17)

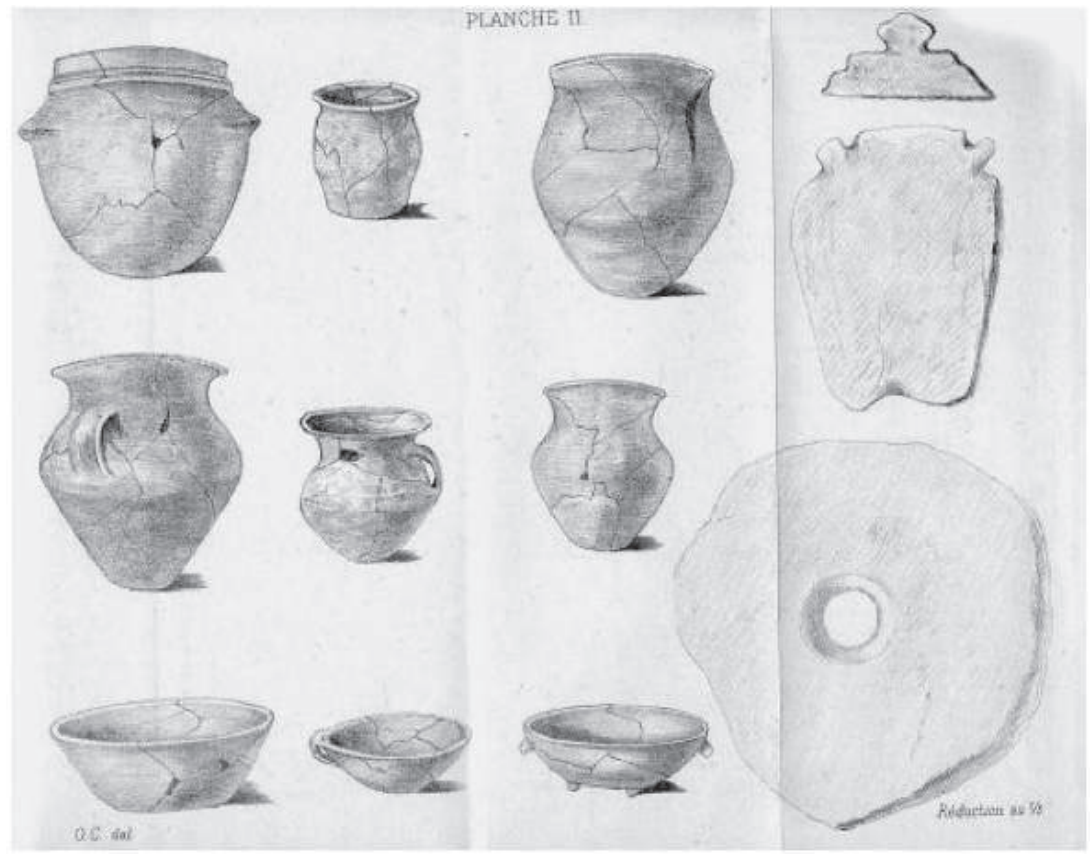

un tournant décisif car durant les décennies suivantes, très peu de fouilles sont menées par la Société Polymathique du Morbihan, à l'exception des travaux de Louis Marsille. Ce dernier est l'un de ceux qui vont largement contribuer à l'installation en 1914 du musée archéologique au Château Gaillard, dont il devient le conservateur en 1920 (fig. 10).

\section{La constitution d'une collection archéologique exceptionnelle}

Les nombreuses fouilles menées par la Société Polymathique du Morbihan durant le XIX ${ }^{\mathrm{e}}$ siècle, ont livré plusieurs milliers d'objets, auxquels se sont ajoutées autant de découvertes isolées (monnaies, haches polies, silex, etc.). Cette collection est venue compléter un autre ensemble déjà bien fourni, celui des sciences naturelles. Dès la fondation de la société savante, en 1826, l'idée de créer un musée est admise dans un souci de conservation des échantillons collectés. Le registre original des séances nous renseigne sur les dons importants reçus durant les toutes premières années ${ }^{40}$.

40. Archives de la Société Polymathique du Morbihan. 
Figure 10 - Membres de la Société Polymathique du Morbihan, dans la cour du musée archéologique au Château Gaillard, à Vannes (Morbihan), en 1926. Au fond, on peut apercevoir la borne milliaire d'Elven (Morbihan), accolée au mur, achetée en 1842

(Centenaire de la Société Polymathique du Morbihan [1826-1926], Vannes, 1927, p. 24)

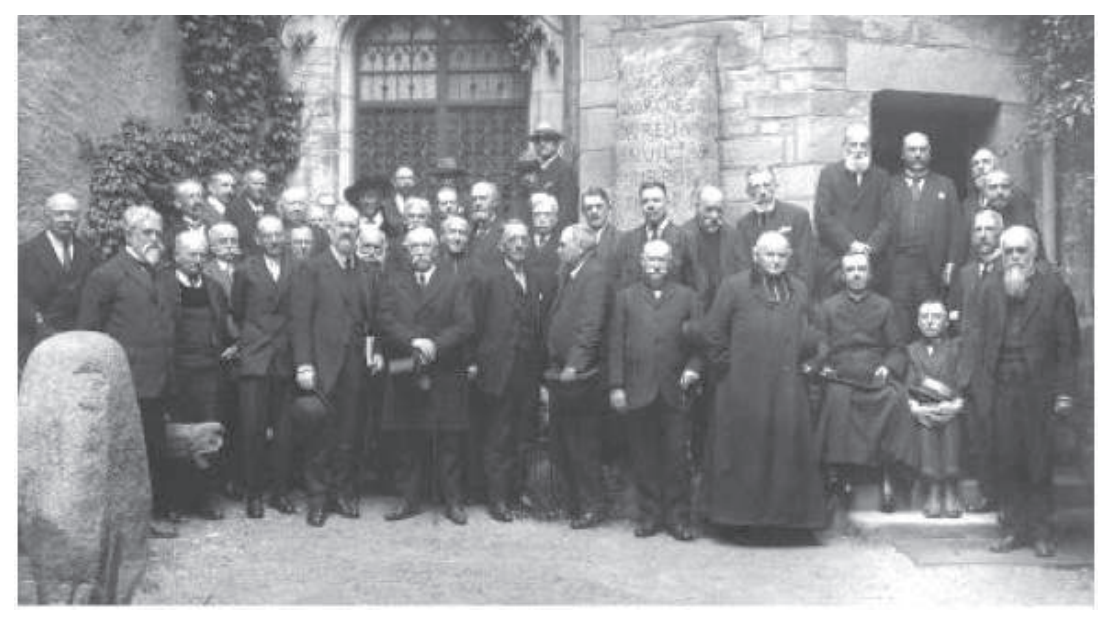

\begin{tabular}{|l|l|}
\hline \multicolumn{1}{|c|}{ Collections entrées entre 1828 et $\mathbf{1 8 3 2}$} & \multicolumn{1}{c|}{ Donateurs } \\
\hline 600 échantillons de minéraux & Collection Jean-Marie Galles \\
\hline Échantillons de minéraux, tous spécifiés & $\begin{array}{l}\text { M. Dubuisson, conservateur du } \\
\text { Muséum de Nantes }\end{array}$ \\
\hline Herbier de 1700 plantes & Collection Jean-Marie Galles \\
\hline Herbier de 1000 plantes (dont 70 espèces d'algues) & Collection M. Le Lièvre \\
\hline Plus de 100 espèces de graminées & M. Lagillardaie \\
\hline 160 espèces d'oiseaux dont 16 oiseaux du Brésil & M. Claret \\
\hline $\begin{array}{l}\text { Plus de } 200 \text { espèces de coquilles, à peu près toutes spécifiées, } \\
\text { et } 50 \text { espèces fossiles }\end{array}$ & $\begin{array}{l}\text { M. Collard des Cherres, membre } \\
\text { de la Société Linnéenne de } \\
\text { Bordeaux }\end{array}$ \\
\hline $\begin{array}{l}\text { Un assez grand nombre d'échantillons de diverses espèces } \\
\text { de zoophytes, quelques mammifères, quadrupèdes, ovipares } \\
\text { et poissons, collection d'insectes dont le nombre excède } \\
\text { celui de tout ce que la Société possède dans toutes les autres } \\
\text { classes réunies }\end{array}$ & Dr Honorat \\
\hline $\begin{array}{l}\text { Plusieurs fascicules d'hydrophytes terrestres et fluviatiles du } \\
\text { département de l'Orne }\end{array}$ & M. Le Lièvre \\
\hline $\begin{array}{l}\text { Un assez grand nombre de productions curieuses de la Médi- } \\
\text { terranée }\end{array}$ & $\begin{array}{l}\text { M. de Bellegarde, directeur des } \\
\text { douanes à Lorient }\end{array}$ \\
\hline $\begin{array}{l}\text { Collections de minéraux, de coquilles et de plantes } \\
\text { Une suite assez nombreuse d'échantillons minéralogiques }\end{array}$ & $\begin{array}{l}\text { Cabinet de curiosité de } \\
\text { M. Le Bourg, de Landerneau } \\
\text { (achat de la Société) }\end{array}$ \\
\hline Don du gouvernement français \\
\hline
\end{tabular}


La mission essentielle de la conservation est remplie par plusieurs personnalités incontournables, dont le travail permet une évaluation quantitative et qualitative précise des collections et ce, par le biais de catalogues d'inventaire.

\begin{tabular}{|c|c|c|}
\hline \multicolumn{3}{|c|}{ Conservateurs des musées de la Société Polymathique (1826-1926) } \\
\hline Dates & Sciences naturelles & Archéologie et Histoire \\
\hline $1826-1828$ & \multicolumn{2}{|l|}{ Luzcot } \\
\hline $1828-1860$ & \multicolumn{2}{|l|}{ Jean-Marie Galles } \\
\hline 1861-1865 & \multicolumn{2}{|l|}{ Amand Taslé } \\
\hline $1866-1877$ & Amand Taslé & \multirow{2}{*}{ Léon Davy de Cussé * } \\
\hline $1878-1879$ & \multirow{2}{*}{ Jules Revelière } & \\
\hline 1880 & & \multirow{6}{*}{ Joseph-Marie Le Mené * } \\
\hline $1881-1883$ & Monteil & \\
\hline $1884-1887$ & Perrin & \\
\hline $1888-1891$ & Jules Taslé & \\
\hline 1892 & Pozzy & \\
\hline 1893-1909 & \multirow{2}{*}{ Leguillon-Guyot } & \\
\hline $1910-1918$ & & \multirow{2}{*}{ Léon Lallement } \\
\hline 1919 & \multirow{2}{*}{ Chabot } & \\
\hline $1920-1926$ & & Louis Marsille * (jusqu'en 1955) \\
\hline
\end{tabular}

* Ces trois conservateurs ont réalisé et publié un catalogue d'inventaire des collections archéologiques, un an après leur entrée en fonction respective.

Si les sciences naturelles on vu défiler nombre de conservateurs, les collections archéologiques, quasiment inexistantes jusqu'au milieu du XIX ${ }^{e}$ siècle, ont bénéficié d'une certaine stabilité. Par la suite, le nombre d'objets issus de fouilles augmente de façon exponentielle. A sa prise de fonction en 1866, Léon Davy de Cussé, conservateur jusqu'en 1879, entreprend un premier inventaire qu'il publie l'année suivante. Il propose une "Description des objets de l'Age de la pierre polie contenus dans le musée $\operatorname{archéologique}^{41}$ ", et se limite donc aux collections du Néolithique. On y distingue les collections appartenant à la société savante (451 numéros d'inventaire) et les dépôts (85 numéros). À eux seuls, les tumulus carnacéens totalisent près de la moitié du catalogue.

En compagnie de Louis Galles, Léon de Cussé participe à la fouille de divers monuments mégalithiques du Morbihan et en assure le relevé des plans. La photographie est encore rare et le dessin reste le meilleur moyen de documenter les vestiges archéologiques (objets ou monuments). C'est une discipline dans laquelle ce polymathe montre un véritable talent. D'une grande patience, on lui confie aussi le soin de reconstituer les réci-

41. Par Davy de Cussé, Léon, Galles, Louis et D’Ault-Dumesnil, Geoffroy, impr. Galles, Vannes, 32 p., 2 pl. 
La Société Polymathique et la naissance de collections archéologiques en Morbihan

\begin{tabular}{|l|c|c|c|l|}
\hline \multicolumn{1}{|c|}{ Tumulus carnacéens découverts entre 1853 et 1864 } \\
\hline Nom du site & $\begin{array}{c}\mathbf{N}^{\circ} \text { catalogue } \\
\mathbf{1 8 6 7}\end{array}$ & $\begin{array}{c}\text { haches } \\
\text { polies }\end{array}$ & $\begin{array}{c}\text { Perles et pende- } \\
\text { loques } \\
\text { (variscite) }\end{array}$ & \multicolumn{1}{|c|}{ Autres éléments } \\
\hline Tumiac (Arzon) & 1 à 42 & 32 & 248 & 5 débris organiques \\
\hline Saint-Michel (Carnac) & 42 à 89 & 36 & 108 & $\begin{array}{l}39 \text { perles en os, des éclats } \\
\text { de silex }\end{array}$ \\
\hline $\begin{array}{l}\text { Mané er Hroeck } \\
\text { (Locmariaquer) }\end{array}$ & $90-217$ & 104 & 50 & $\begin{array}{l}1 \text { anneau-disque, des éclats } \\
\text { de silex, 1 cristal de quartz }\end{array}$ \\
\hline Le Moustoir (Carnac) & $226-241$ & 0 & 1 & $\begin{array}{l}2 \text { pendeloques en ser- } \\
\text { pentine, 5 lames en silex, } \\
4 \text { vases en terre cuite, } 1 \\
\text { boule en calcaire, des éclats } \\
\text { de silex }\end{array}$ \\
\hline
\end{tabular}

pients en terre cuite et autres mobiliers pour en restituer la forme initiale. Il réalise aussi des fac-similés d'objets archéologiques régionaux ou exotiques que le musée de Vannes ne possède pas. Son talent se révèle le plus dans les nombreuses planches aquarellées d'objets qu'il réalise et que conserve aujourd'hui la bibliothèque de la Société Polymathique du Morbihan $^{42}$ (fig. 11, 13 et 14). Dans les dernières années de sa vie, deux de ses fils, Henri et Fernand, participent à ses fouilles et prennent ensuite le relais dans la recherche archéologique en Morbihan. Ces deux générations de chercheurs ont offert au musée de Vannes plus de 200 objets de toutes les époques et laissent derrière eux une importante documentation scientifique. Voici un extrait des paroles prononcées aux obsèques de Léon de Cussé, le 24 juillet 1886 : "De Cussé, nul de nous ne l'ignore, était un artiste remarquable que sa modestie seule garda dans une relative obscurité. Les procédés des meilleures écoles n'avaient pour lui ni difficultés ni secrets; soit qu'il eût à reproduire à grands traits des effets dont il savait pressentir les impressions lointaines, soit qu'il eût à rendre, dans des aquarelles d'un fini admirable, les reflets d'une gemme, les détails les plus délicats d'une arme ou d'un bijou ${ }^{43}$."

En 1880, l'abbé Joseph-Marie Le Mené devient le nouveau conservateur et publie l'année suivante, le premier catalogue du musée archéologique ${ }^{44}$. Bien que considéré comme exhaustif, cet inventaire affiche des défauts de méthodologie importants : il ne reprend pas la numérotation initiale attribuée en 1867. De plus, la nouvelle numérotation n'est pas continue et se répète, en s'appuyant notamment sur un jeu d'étiquettes de couleurs. Un simple comptage du médaillier figure en fin de catalogue.

42. Ces aquarelles d'objets sont aujourd'hui réunies dans deux grands albums reliés, l'un intitulé "Dolmens du Morbihan " (63 pl. dont 7 manquantes) et l'autre "Les bronzes du Morbihan " (24 pl.).

43. BSPM, 1886, 30, p. 1-2.

44. Impr. Galles, Vannes, 1881, 72 p. et 3 pl. 
Figure 11 - Haches polie du tumulus Saint-Michel, à Carnac (Morbihan)

(aquarelle de Léon de Cussé, vers 1870-1880, @ Archives de la Société Polymathique du Morbihan)

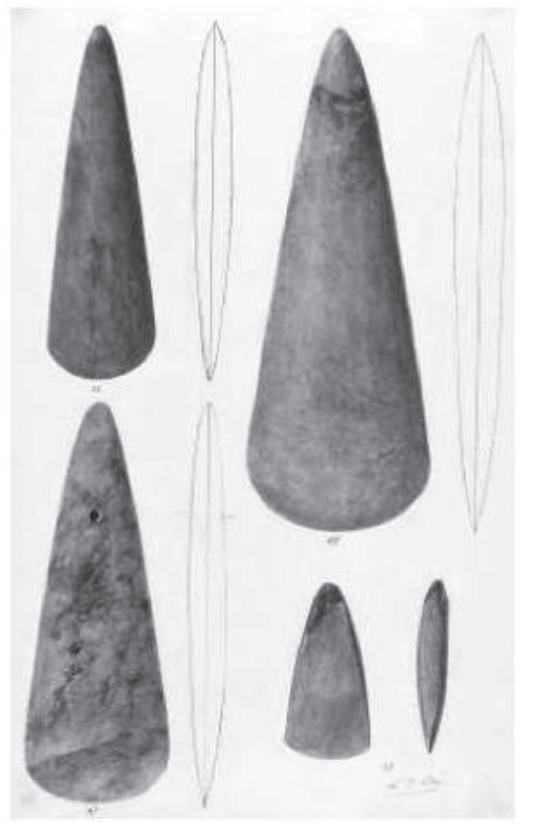

Figure 12 - Caveau funéraire de Mané er Hroeck (Locmariaquer, Morbihan)

(plan aquarellé, non signé, XIX siècle, (c) Manuscrits de la Société Polymathique du Morbihan)
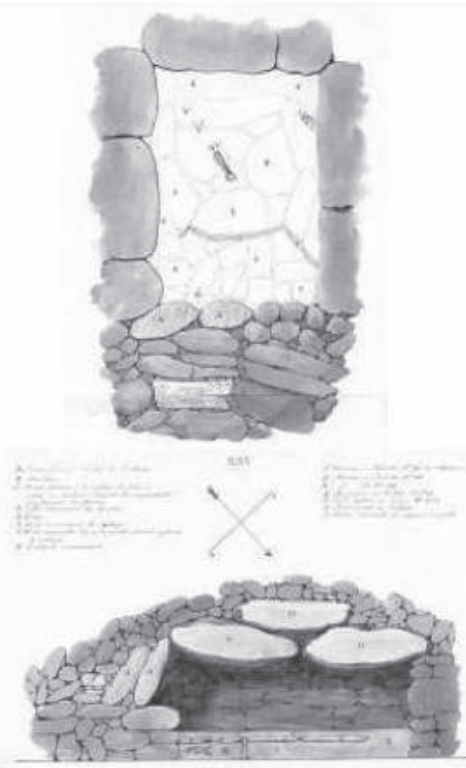

\begin{tabular}{|c|c|c|c|c|}
\hline \multicolumn{5}{|c|}{ Catalogue archéologique de 1881} \\
\hline \multirow[b]{2}{*}{ Nature de la collection } & \multicolumn{2}{|c|}{$\mathrm{N}^{\circ}$ dans le catalogue } & \multirow{2}{*}{$\begin{array}{l}\text { Nb de } \\
\text { numéros }\end{array}$} & \multirow[b]{2}{*}{ Total } \\
\hline & $\begin{array}{l}\text { Propriété Société Poly- } \\
\text { mathique du Morbihan }\end{array}$ & Objet en dépôt & & \\
\hline Néolithique & 1 à 772 & 1 à 54 & 826 & \multirow{5}{*}{1602} \\
\hline Objets exotiques & 1 à 92 & 0 & 92 & \\
\hline $\begin{array}{l}\text { Âge du bronze, âge du fer, } \\
\text { Gallo-romain }\end{array}$ & 1 à 400 & 1 à 22 & 422 & \\
\hline $\begin{array}{l}\text { Moyen Âge, Renaissance, } \\
\text { Époque moderne }\end{array}$ & 1 à 221 & 0 & 221 & \\
\hline Gros objets & 1 à 41 & 0 & 41 & \\
\hline $\begin{array}{l}\text { Monnaies gauloises } \\
\text { Monnaies grecques } \\
\text { Monnaies romaines } \\
\text { Monnaies bretonnes } \\
\text { Monnaies françaises } \\
\text { Monnaies baronniales } \\
\text { Monnaies étrangères } \\
\text { Jetons et médailles } \\
\text { Pièces non classées }\end{array}$ & $\begin{array}{c}34 \\
77 \\
2000 \\
184 \\
700 \\
350 \\
180 \\
140 \text { et } 340 \\
300\end{array}$ & 0 & \multicolumn{2}{|c|}{$\begin{array}{l}\text { Total du médaillier : } \\
\qquad 4305\end{array}$} \\
\hline
\end{tabular}


Figure 13 - Vase-support du tumulus du Moustoir ou Er Mané (Carnac, Morbihan)

(esquisse de Léon de Cussé, vers 1870-1880,

(C) Archives de la Société Polymathique du Morbihan)

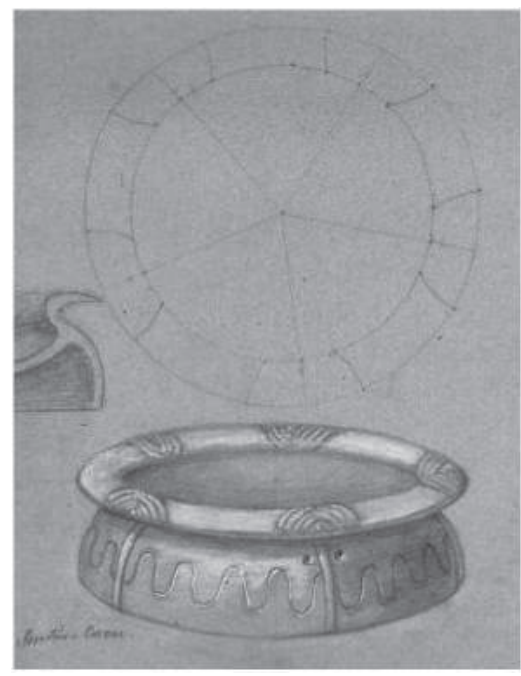

Figure 14 - Vases du tumulus de Kercado (Carnac, Morbihan)

(aquarelle de Léon de Cussé, vers 1870-1880,

(C) Archives de la Société

Polymathique du Morbihan)

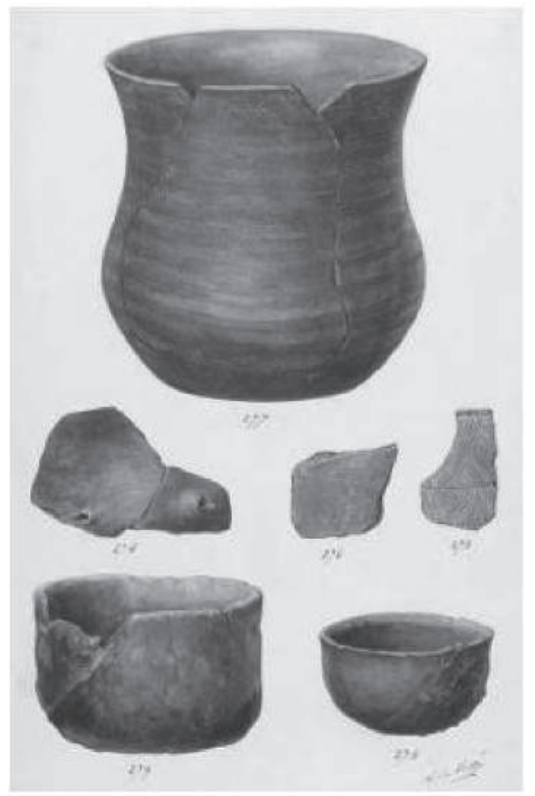

En cette fin de XIX ${ }^{\mathrm{e}}$ siècle, l'archéologie s'est aussi quelque peu démocratisée et les fouilles sont menées de plus en souvent par des instituteurs ou des historiens locaux. Il découle de ce constat que les objets recueillis alimentent généralement des collections privées et le marché des antiquaires. Fort heureusement, la Société Polymathique conserve encore un important réseau de membres à travers tout le département et parvient à sauver des découvertes archéologiques, parfois en les achetant. Le musée archéologique de Vannes continue donc de s'enrichir de beaux ensembles :

- 1888 : mise au jour fortuite du trésor gaulois de Brec'h, composé de parures en verre, en or et en cuivre et de monnaies tardives des Vénètes ${ }^{45}$;

- 1892 : Jules Revelière découvre le célèbre poignard gaulois de Kernavest (Quiberon) ${ }^{46}$. Cet objet sera donné au musée de la Société Polymathique du Morbihan par la famille Revelière, en 1963 (fig. 15);

45. LE MENÉ, Joseph-Marie, " Une trouvaille à Brec'h », BSPM, 1896, 40, p. 154-155.

46. BSPM, 1894, 38, p. 157-166. 
- 1893 : les polymathes explorent le théâtre antique de Locmariaquer et trouvent une trentaine d'objets ${ }^{47}$;

- 1899-1901 : lors des travaux d'aménagement du cimetière de Boismoreau à Vannes, est récolté un imposant mobilier antique comprenant 75 objets ou fragments ${ }^{48}$;

- 1902 : découverte d'un coffre de pierre sous tumulus à Belle-Île-en$\operatorname{Mer}^{49}$ (fig. 16);

- 1914 : le comte de Lantivy et Jules de La Martinière fouillent l'atelier de potiers carolingien de Meudon à Vannes ${ }^{50}$.

\section{Figure 15 - Poignard gaulois de Kernavest à Quiberon \\ (Morbihan), découvert par Jules Revelière en 1892 \\ (BSPM, 38, 1894)}

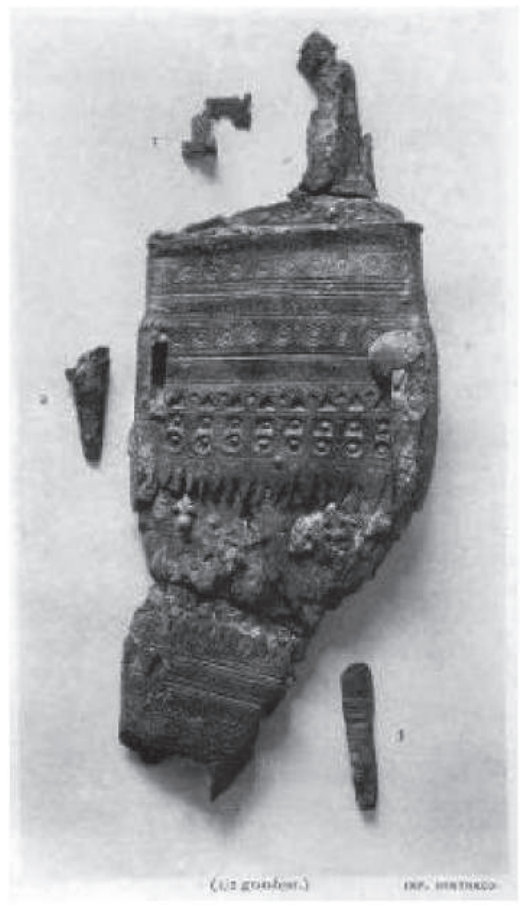

Figure 16 - Objets découverts en 1896 à Belle-Île-en-Mer (Morbihan) (BSPM, 46, 1902)

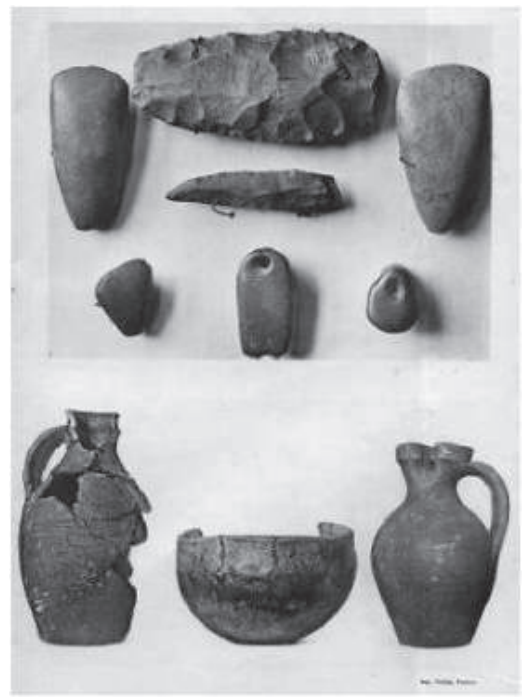

47. Closmadeuc, Gustave de, "Théâtre romain de Locmariaquer ", BSPM, 1893, 37, p. 181192.

48. Lallement, Léon et Aveneau De La Granclère, Paul, "Fouilles pratiquées au nouveau cimetière ", BSPM, 1900, 44, p. 28-39.

49. Closmadeuc, G. de, "Découverte de cists tumulaires à Belle-Île, en 1896 ", BSPM, 1902, 46, p. 305-309.

50. " Les poteries décorées de Meudon (près Vannes, Morbihan) ", RA, 1914, 2, p. 67. 
En 1910, Léon Lallement succède à l'abbé Joseph-Marie Le Mené à la direction du musée archéologique et, rapidement, se pose à nouveau la question de trouver un local pour accueillir les collections. En effet, depuis près de 60 ans, le musée était entassé dans la maison Lorvol, une demeure devenue vétuste située 8 place des Lices, où la société savante devait payer un loyer (fig. 17). Lors d'une séance extraordinaire tenue le 21 mars 1912, la majorité des polymathes présents accepte le principe d'acheter un grand logis du XV e siècle : Château Gaillard. Seul le conservateur du musée est hostile à cet achat car il redoute un nouveau transport des précieuses collections. L'hôtel particulier est finalement acheté le 20 juillet 1912 par un financement multiple, mais surtout assuré à plus de 70 \% par des souscriptions de sociétaires. Après d'importants travaux de restaurations et d'aménagement intérieur, le déménagement des objets débute au printemps $1914^{51}$.

Louis Marsille (1872-1966) ${ }^{52}$ adhère à la Société Polymathique en 1908 et il y fait la connaissance d'illustres érudits : le docteur de Closmadeuc et le chanoine Le Mené. Bourreau de travail, il est l'auteur de plus d'une centaine

Figure 17 - Salle consacrée au Moyen Âge, dans l'ancien musée archéologique (maison Lorvol), ì Vannes (Morbihan) (cl. du R.P. de Gouttepagnon (fin du XIX siècle?), (c) Manuscrits de la Société Polymathique du Morbihan)

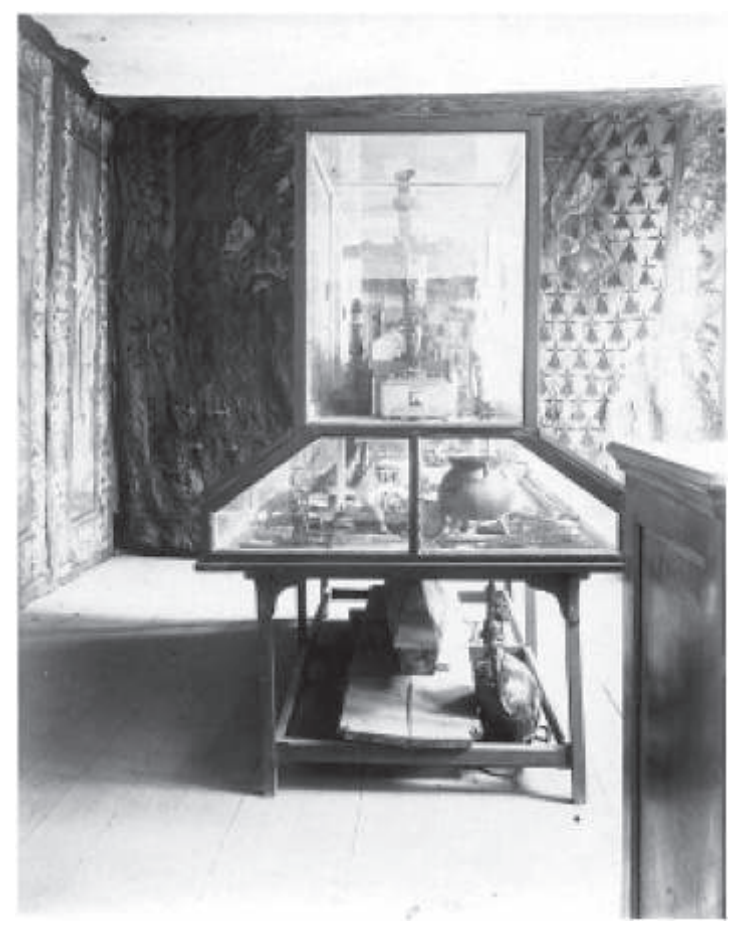

51. Centenaire de la Société Polymathique du Morbihan (1826-1926), op. cit., p. 170-174.

52. Né à Lorient, d'un père industriel, le jeune Louis âgé seulement de 8 ans grattait déjà dans les ruines d'une villa romaine située proche de leur maison de Carnac. Après de longues études qui le mènent jusqu'à la thèse, il devient docteur en droit. 
d'articles d'histoire ou d'archéologie, consacrés, entre autres, aux objets de l'âge du Bronze, à l'époque gallo-romaine et aux monuments mégalithiques. Il devient conservateur du musée archéologique en 1920 et ce, jusqu'en 1955. Dans la tradition de ses prédécesseurs, il consacre sa première année de fonction à la rédaction d'un nouveau catalogue d'inventaire ${ }^{53}$. Il décide, à juste titre, une refonte totale de l'inventaire précédent (1881) pour instaurer une numérotation unique et continue, après avoir classé l'ensemble des collections selon un ordre globalement chronologique et thématique. Cet ouvrage demeure aujourd'hui encore une référence pour la gestion d'une partie des collections du musée de Vannes. En 1925, il découvre et étudie un site néolithique, peut-être de la civilisation du Campignien, qui livre des centaines d'outils en grès (percuteurs, racloirs, haches, tranchets, etc.) ${ }^{54}$. Au cours de sa vie, il donne de nombreux objets et son importante collection archéologique personnelle entre au musée en 1966, totalisant aujourd'hui plus de 300 numéros d'inventaire.

\begin{tabular}{|c|c|c|c|}
\hline \multicolumn{4}{|c|}{ Catalogue archéologique de 1921} \\
\hline Nature de la collection & $\mathbf{N}^{\circ}$ dans le catalogue & Nombre de $n^{\circ}$ & Total \\
\hline Néolithique & 1 à 1203 & 1203 & \multirow{12}{*}{$\begin{array}{c}3156 \\
\text { numéros }\end{array}$} \\
\hline Protohistoire & 1204 à 1217 & 14 & \\
\hline Âge du Bronze & 1218 à 1554 & 337 & \\
\hline Âge du Fer & 1555 à 1713 & 159 & \\
\hline Gallo-romain & 1714 à 2085 & 372 & \\
\hline Moyen Âge & 2086 à 2090,2606 à 2627 & 27 & \\
\hline Antiquités méditerranéennes & 2091 à 2168 & 78 & \\
\hline Extra-européen & 2169 à 2227,2467 à 2605 & 198 & \\
\hline Préhistoire (hors Bretagne) & 2228 à 2466 & 239 & \\
\hline Histoire, divers & 2628 à 3029bis & 403 & \\
\hline Musée lapidaire & 3030 à 3090 & 61 & \\
\hline Supplément (janvier 1921) & 3091 à 3156 & 66 & \\
\hline
\end{tabular}

Ainsi, entre 1881 et 1921, le nombre de numéros attribués aux collections archéologiques et historiques a quasiment doublé, passant de 1602 à 3156. Ce dernier numéro ne reflète en rien le nombre réel d'objets listé dans l'inventaire Marsille, qui est nettement plus important et doit avoisiner les 3700. Quant au médaillier, pour lequel il n'existe pas d'inventaire exhaustif, le nombre de monnaies, médailles et jetons est estimé entre 5000 et 8000 . Les collections du musée de la Société Polymathique ne se limitent pas à l'archéologie mais comprennent également de nombreux spécimens natu-

53. Catalogue du Musée archéologique de la Société Polymathique du Morbihan, Impr. Galles, Vannes, 1921, 177 p.

54. MARSILLE, Louis, "L'atelier néolithique de Nazareth en Saint-Congard (Morbihan) ", $B S P M, 1925,64$, p. 3-9. LÉJARDS, Jacques, " Outillage en grès armoricain, ferme de Nazareth en Saint-Congard (Morbihan) ", BSPM, 1966, 93, p. 8-17. 
rels, la plupart collectés avant 1930 : 300 liasses d'herbiers (soit environ 10000 plantes), 6000 échantillons de roches et de minéraux, 2000 fossiles, 1000 animaux naturalisés, 15000 coquillages, des coraux et des œufs d'oiseaux.

Au cours du xx $x^{\mathrm{e}}$ siècle, les dons d'objets archéologiques, historiques et extra-européens demeurent importants. Pour les collections entrées après l'inventaire de Louis Marsille (c'est-à-dire entre 1921 et 2000), plus de 2700 nouvelles notices d'objets ont été ainsi ajoutées dans l'actuel inventaire informatisé ${ }^{55}$. L'année 2000 marque enfin un nouveau tournant dans l'histoire du musée archéologique, par la signature d'une convention entre la Ville de Vannes et la Société Polymathique du Morbihan ${ }^{56}$ : les collections, gérées par le musée municipal, bénéficient des nouvelles technologies (informatisation des données, images numériques...) et des règles de la conservation préventive, nécessaires à leur préservation et leur valorisation auprès des publics.

La Société Polymathique du Morbihan, qui fêtera son bicentenaire en 2026, est une institution toujours active aujourd'hui. Aux cours du XIX et au début du $\mathrm{xx}^{\mathrm{e}}$ siècle, cette société savante a joué un rôle précurseur et fondamental dans la recherche archéologique. Par les nombreuses publications de ses découvertes et la constitution d'une documentation scientifique unique, elle offre aux chercheurs actuels une source d'information inépuisable. La connaissance des vestiges archéologiques du Morbihan serait infiniment moindre, à n'en pas douter, sans l'héritage de ces générations d'érudits et historiens, animés de la passion pour les sciences humaines. Par une démarche collégiale et une volonté de conserver pour transmettre, cette société savante a bien souvent évité la dispersion des objets exhumés lors de fouilles, et assuré la collecte d'innombrables trouvailles isolées faites dans le département. Cette collection archéologique, par son ampleur, par sa diversité et par la présence d'objets exceptionnels, demeure encore certainement l'une des plus riches et des plus belles de Bretagne.

55. Le Pennec, Christophe, « Premier bilan de l'inventaire des collections archéologiques de la Société Polymathique du Morbihan ", BSPM, 2005, CXXXI, p. 15-28.

56. Le SAUX, Marie-Françoise et Frelaut, Bertrand, La Société Polymathique du Morbihan, février 2005, 48 p. 


\section{RÉSUMÉ}

La création de la Société Polymathique du Morbihan (société savante), en 1826, constitue un évènement fondateur pour la recherche scientifique dans le département. D'abord limités à la collecte d'échantillons de sciences naturelles jusque vers 1850, les travaux de ces érudits se concentrent ensuite sur les fouilles archéologiques et permettent la découverte de plus d'une centaine de sites ou monuments, toutes époques confondues. Il en découle la création d'un musée archéologique en 1853 et la constitution d'une exceptionnelle collection, composée d'environ 45000 objets, spécimens ou échantillons, parmi les plus belles de Bretagne.

\section{ABSTRACT}

The setting up of the Sociéte Polymathique du Morbihan (learned society), in 1826, was a seminal event in the scientific research of the département. At first confined to the collecting of natural science samples until around 1850, the works of these scholars were then focused on archaeological excavations and enabled the discovery of over one hundred sites or monuments, from all eras. This resulted in the founding of an archaeological museum in 1853 and the constitution of an exceptional collection, composed of around 45,000 objects, specimens and samples, which are among the most beautiful that can be found in Brittany. 


\title{
La sculpture sur pierre de l'époque romaine et de l'âge du Fer en Bretagne : des collections embryonnaires avant 1941
}

\author{
Jean-Yves ÉvEILLARD \\ Université de Bretagne occidentale, \\ Centre de recherche bretonne et celtique
}

Dans le cadre de la deuxième journée d'histoire de l'archéologie du Grand Ouest, notre communication a porté sur la sculpture sur pierre (statuaire et reliefs) de l'époque romaine ainsi que du second âge du Fer connue en Bretagne avant la loi de 1941. Le point de départ obligé d'une telle enquête est le Recueil d'Émile Espérandieu dont le dernier volume avant les suppléments ajoutés par Raymond Lantier est paru en 1938, soit à une date très voisine du terminus fixé ${ }^{1}$. Dans les cinq départements bretons Espérandieu ne recense que 34 pièces. De ce chiffre nous en avons retiré quelques unes, fausses ou mal identifiées (par exemple la Vénus de Quinipily, sur laquelle nous reviendrons cependant), ainsi que trois plaques de schiste en relief méplat, un type d'objet commun parmi les découvertes en Bretagne mais que nous hésitons à classer dans la sculpture à proprement parler $^{2}$. À l'inverse, nous avons ajouté plusieurs stèles funéraires de Nantes qu'Espérandieu ne prend pas en compte en raison, semble-t-il, de l'absence de motifs figurés à l'intérieur du décor architectural, décor qui nous semble être cependant un critère suffisant pour que soient retenues lesdites stèles. Nous arrivons alors à un total de 42 . Ce chiffre, modeste comparé à beaucoup de régions de la Gaule romaine comme la Narbonnaise, l'Aquitaine, la Bourgogne ou la Rhénanie, ne dépasse pas le quart d'un dénombrement actualisé qui avoisine les 165 unités pour l'âge du Fer et l'Époque romaine réunis. D'où il ressort qu'en Bretagne la plus grande partie des sculptures sur pierre pour ces deux périodes a été connue dans la seconde moitié du $\mathrm{xx}^{\mathrm{e}}$ siècle. Dans de telles conditions

1. ESPÉRANDIEU, Émile, Recueil des bas-reliefs, statues et bustes de la Gaule romaine, Paris, 1907-1938, 11 vol.

2. LANGOUËT, Loïc et QuESNEL, Laurent, « Les plaques décorées en schiste de la Bretagne armoricaine sous l'Empire romain ", $R A O, 17,2000$, p. 215-237. 